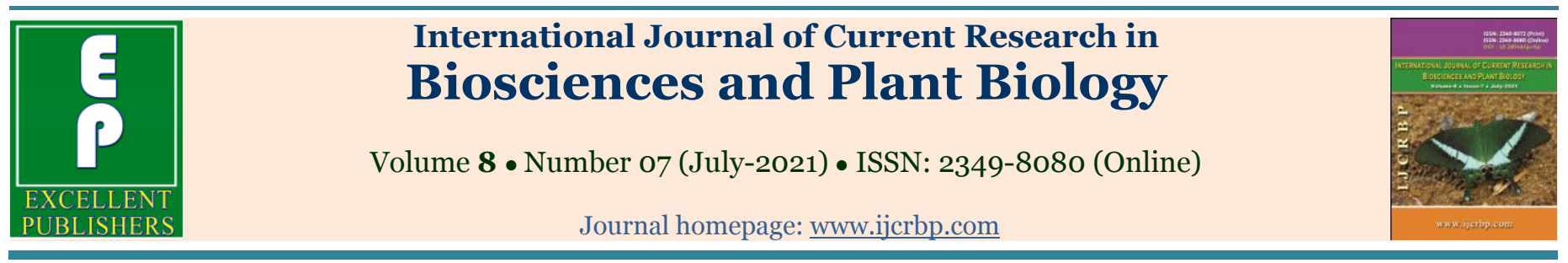

\title{
Ten species of liverworts, addition to Bryoflora of Andhra Pradesh, India
}

\author{
Ananthaneni Sreenath and Boyina Ravi Prasad Rao
}

Biodiversity Conservation Division, Department of Botany, Sri Krishnadevaraya University, Ananthapuramu -515003, Andhra Pradesh, India

*Corresponding author; e-mail: biodiversityravi@gmail.com

\begin{tabular}{ll}
\hline Article Info & Abstract \\
\hline Keywords: & Ten species of liverworts viz., Exormotheca tubifera Kashyap, Fossombronia himalayensis Kashyap, \\
Bryoflora new records & Cheilolejeunea intertexta (Lindenb.) Stephani, C. krakakammae (Lindenb.) R.M. Schust., \\
Cheilolejeunea & Jungermannia appressifolia Mitt., J. macrocarpa Stephani, J. rubripunctata (S. Hatt.) Amakawa, \\
Exormotheca & Plagiochila beddomei Steph., P. chinensis Stephani and P. fruticosa Mitt., collected from different \\
$\begin{array}{l}\text { Fossombronia } \\
\text { Jungermannia }\end{array}$ & localities of the state are reported as new distributional records to Andhra Pradesh. \\
Plagiochila & \\
\hline & \\
\hline
\end{tabular}

\section{Introduction}

Andhra Pradesh is the seventh largest state in Indian union, it covers an area about 162,970 sq. kilometers and lies between $12^{\circ} 37^{\prime}$ and $19^{\circ} 25^{\prime} \mathrm{Nl}$ and $76^{\circ} 45^{\prime}$ and $84^{\circ} 72^{\prime}$ El (Fig. 1). The state comprises 13 districts, there are two areas namely called Rayalaseema and Costal Andhra. The state is having about 17.86 percentage of forest cover (FSI, 2019), major portion of Eastern Ghats hill ranges, it is also having different types of vegetation's, nearly more than 180 waterfalls (seasonal and perennial) and different types of altitudinal variations from sea level to 1690 meters of above mean sea level.

Owing the presence of rich bryophyte habitats especially moist shady interior forest habitats, and nearly around 180 water falls (seasonal and perennial) in Andhra Pradesh, we held explorations for bryophytes during 2016 -2020. During the survey, we could collect some curious liverwort specimens from various localities of Eastern Ghats of Andhra Pradesh. After critical microscopic examination, these specimens were identified as liverwort species viz., Exormotheca tubifera Kashyap, Fossombronia himalayensis Kashyap, Cheilolejeunea intertexta (Lindenb.) Stephani, Cheilolejeunea krakakammae (Lindenb.) R.M. Schust., Jungermannia appressifolia Mitt., J. macrocarpa Stephani, J. rubripunctata (S. Hatt.) Amakawa, Plagiochila beddomei Steph., P. chinensis Stephani and P. fruticosa Mitt.

Perusal of literature (Bapna and Kachroo. 2000; Dandotiya et al., 2011; Sandhya Rani et al., 2014; Sreenath and Ravi Prasad Rao, 2019; Sreenath and Ravi Prasad Rao, 2020a and 2020b; Sreenath et al., 
2020a and 2020b; Asthana and Srivastava, 2020) revealed that these species are nowhere recorded in the state of Andhra Pradesh till date and thus forms new distributional records to the state.

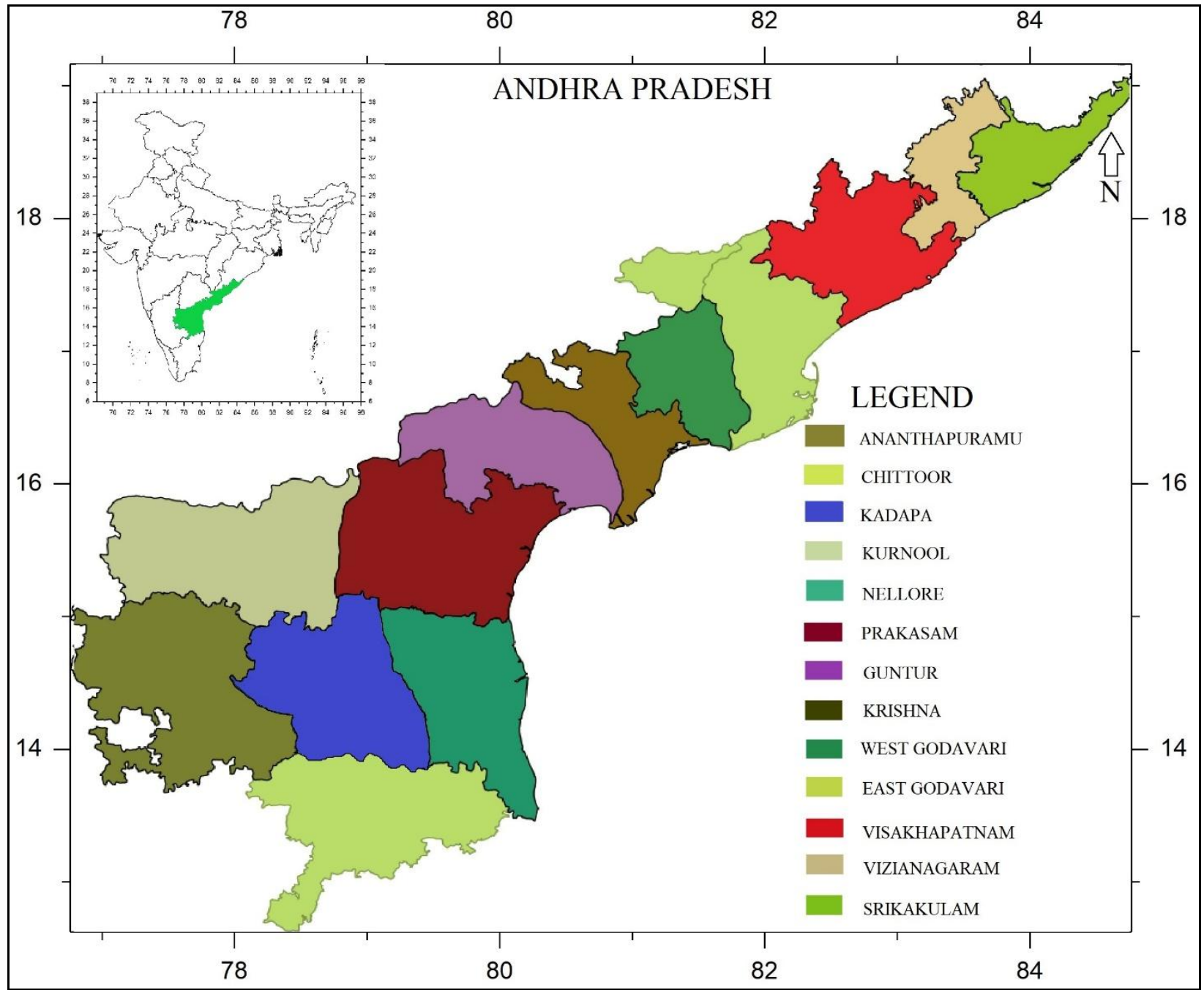

Fig. 1: Map of Andhra Pradesh.

\section{Materials and methods}

Extensive field explorations were conducted during 2016 - 2020 in the state of Andhra Pradesh covering all the seasons. The collected specimens labeled with field number were air-dried and preserved in thick paper covers. Observations were made pertaining to habitat and filed photos were taken using Nikon D3300. Critical study was done by separating plant parts using micro forceps and observed Olympus $\mathrm{CH} 2 \mathrm{Oi}$ and Olympus SZ61) microscopes. Measurements were taken using ERMA ocular micrometer. Microscopic photos were taken by using Moto g3 turbo and Samsung on6 equipped with $13 \mathrm{MP}$ cameras. Identification of the specimens was done by using standard floras. In this paper, citation, description, habitat details, voucher specimen data, and field photographs were provided for the taxa. All the specimens were deposited in the herbarium of Sri Krishnadevaraya University, Ananthapuramu. Abbreviations for the plant collectors are: AS (Ananthaneni Sreenath) and BR (Boyina Ravi Prasad Rao).

\section{Results}

\section{Systematic treatment}

Exormotheca tubifera Kashyap, New Phytologist 13: 208. 1914; Bryophytes of Kerala, Liverworts 1: 34. 2017. (Fig. 2 A-B). 

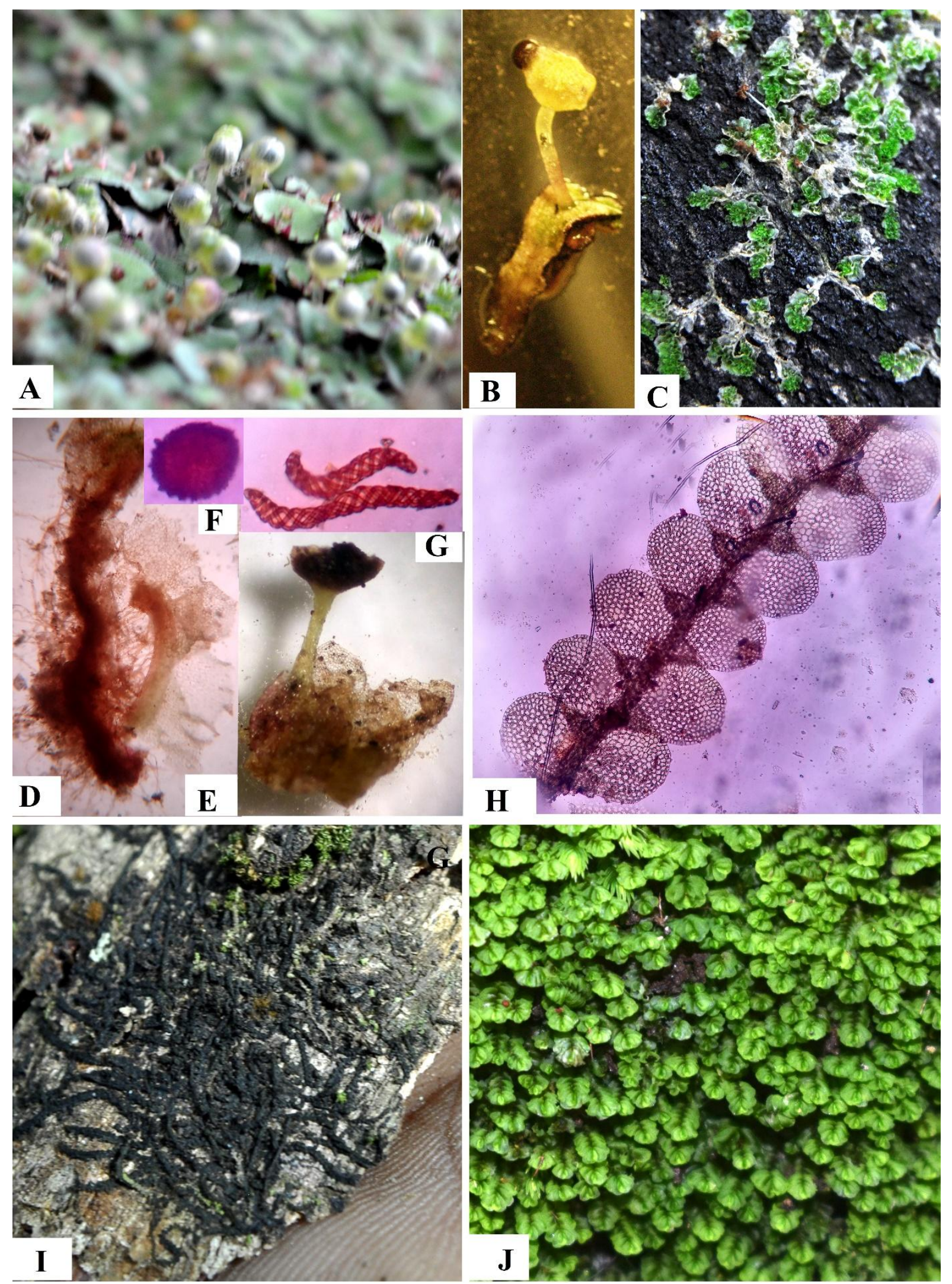

Fig. 2: A-B. Exormotheca tubifera, C-G. Fossombronia himalayensis H. Cheilolejeunea intertexta, I. Cheilolejeunea krakakammae and J. Jungermannia appressifolia. 
Plants thalloid, terricolous, thalli dark greenish, 1-2, dichotomously branched, with prominent midrib, air chambers in one row with simple 3-5 celled assimilatory filaments. Scales in two rows, simple without appendage, antheridia embedded in the thallus, very close behind the female receptacle along the middorsal line in 2-3 rows; female receptacle apical at the dichotomous notch, involucres; sporophyte with short foot, seta and spherical capsule, capsule dark brownish coloured when mature, dehiscence 4-5 valvular. Elaters simple to branched.

Habitat and ecology: Terricolous on moist soils along pathways in moist deciduous forests, associated with Plagiochasma appendiculatum and other mosses.

Distribution: World: India: Himachal Pradesh, Kerala, Madhya Pradesh, Maharashtra, North Western Himalayas and Tamil Nadu.

Specimens examined: India, Andhra Pradesh, Chittoor District, Horsley hills, roadside near fourth turn, 23 August 2016, 51620, BR \& AS.

Fossombronia himalayensis Kashyap, New Phyt. 14: 4. 1915; F. levieri St., Sp. Hep., 6: 4. 1917; Liverworts W. Himalayas \& Punjab Plains, 2: 92 - 93.1932 (Fig. $1 \mathrm{C}-\mathrm{G})$.

Plants thalloid, yellowish-green to green, main stem creeping, 2 - 3 times branched, branches up to $6 \mathrm{~mm}$ long. Rhizoids generally violet, sometimes hyaline, leaves wavy, ascending, overlapping to about $1 / 3$ of the length, outer margin irregularly and indistinctly toothed. Monoecious or dioecious, perianth with wavy margin, sometimes split to the base along one side. Sporophyte stalked, seta up to $5 \mathrm{~mm}$ long, often very short. Capsule usually excreted. Cells of the outer layer of the capsule wall with thin walls, those of the outer layer with U-shaped bands on radial walls at another place. Dehiscence by separation of the apical portion. Spores brown with furcate high lamellae, sometimes forming a few reticulations, rounded, $40-$ $50 \mu \mathrm{m}$ in diameter. Elaters brown, laxly bi- or trispiral, $100-140 \mu \mathrm{m}$ long.

Habitat and ecology: Rupicolous or terricolous on moist rocks, monodominant, or sometimes associated with Reboulia hemispherica (Aytoniaceae) and liverworts.
Distribution: Endemic to India: E. Himalayas, Himachal Pradesh, Karnataka, Madhya Pradesh, Maharashtra, Tamil Nadu, Uttarakhand, West Bengal and Western Himalaya.

Specimens examined: India, Andhra Pradesh, Nellore District Penchalakona hill tops, 07 February 2017, 52273A, AS.

Cheilolejeunea intertexta (Lindenb.) Stephani, Bull. Herb. Boiss 5: 79. 1897; Mazut., J. Hattori Bot. Lab. 30: 172. 1967; G. Asthana et al., Lindbergia 20: 127. 1995; Bapna \& Kachroo, Hepat. India 2: 220. 2000. Lejeunea intertexta Lindenb. Gottesche et al., Syn. Hepat. 379. 1845. Cheilolejeunea kurzii Steph., Bot. Gazette 15: 284. 1890. C. katschalliana Steph., Hedwigia 34: 242. 1895. C. grandiflora Steph., Hepat. 5: 664. 1914. C. subrotunda Herzog, J, Hattori Bot. Lab. 14: 48. 1955; Manju, Eco-sys. Stud. on bryophytes of Wayanad 125 126. 2005 (Fig. $2 \mathrm{H}$ ).

Plants leafy, light greenish, $8-10 \mathrm{~mm}$ long, pinnately branched, branching Radula-Jubula type; leaves imbricate, apex rounded, margin entire, $0.06 \times 0.06$ $\mathrm{mm}$; cells hexagonal, trigones absent, intermediate nodular thickenings absent, leaf marginal cells $7-10$ $\mu \mathrm{m}$ in diameter, leaf middle cells $20-25 \times 15-19 \mu \mathrm{m}$, basal cells small up to $20 \mu \mathrm{m}$ in diameters diagonally; leaf lobules small, $0.10-0.15 \times 0.6-0.9 \mathrm{~mm}$, constricted at apex, tooth unicellular; under-leaves distant, twice as wide as stem, bilobed, margin entire, transversely to obliquely inserted. Plants monoecious. Male inflorescence terminal, on short or long lateral branches, capitate, sometimes intercalary on main axis with $2-5$ pairs of densely imbricate bracts; bracteoles only at base. Female inflorescence on long or short lateral branches with one sub-floral innovations; lobes of bracts $0.35-0.50 \times 0.20-0.25 \mathrm{~mm}$, rounded at apex, margin entire; lobules $0.25-0.30 \mathrm{~mm}$, ovate, 2lobed at apex. Perianth $0.65-0.70 \times 0.45-0.55 \mathrm{~mm}$, obovate, 4-plicate with 2 lateral and 2 ventral side. Sporophyte not seen.

Habitat and ecology: Corticolous on Caryota urens, in evergreen forest in coffee plantation, associated with Erpodium biseriatum.

Distribution: World: Japan, Java, Malaya, Philippines, Samoa, Sri Lanka, Sumatra, Tahiti and India: Andaman and Nicobar Irelands, Kerala and Tamil Nadu. 

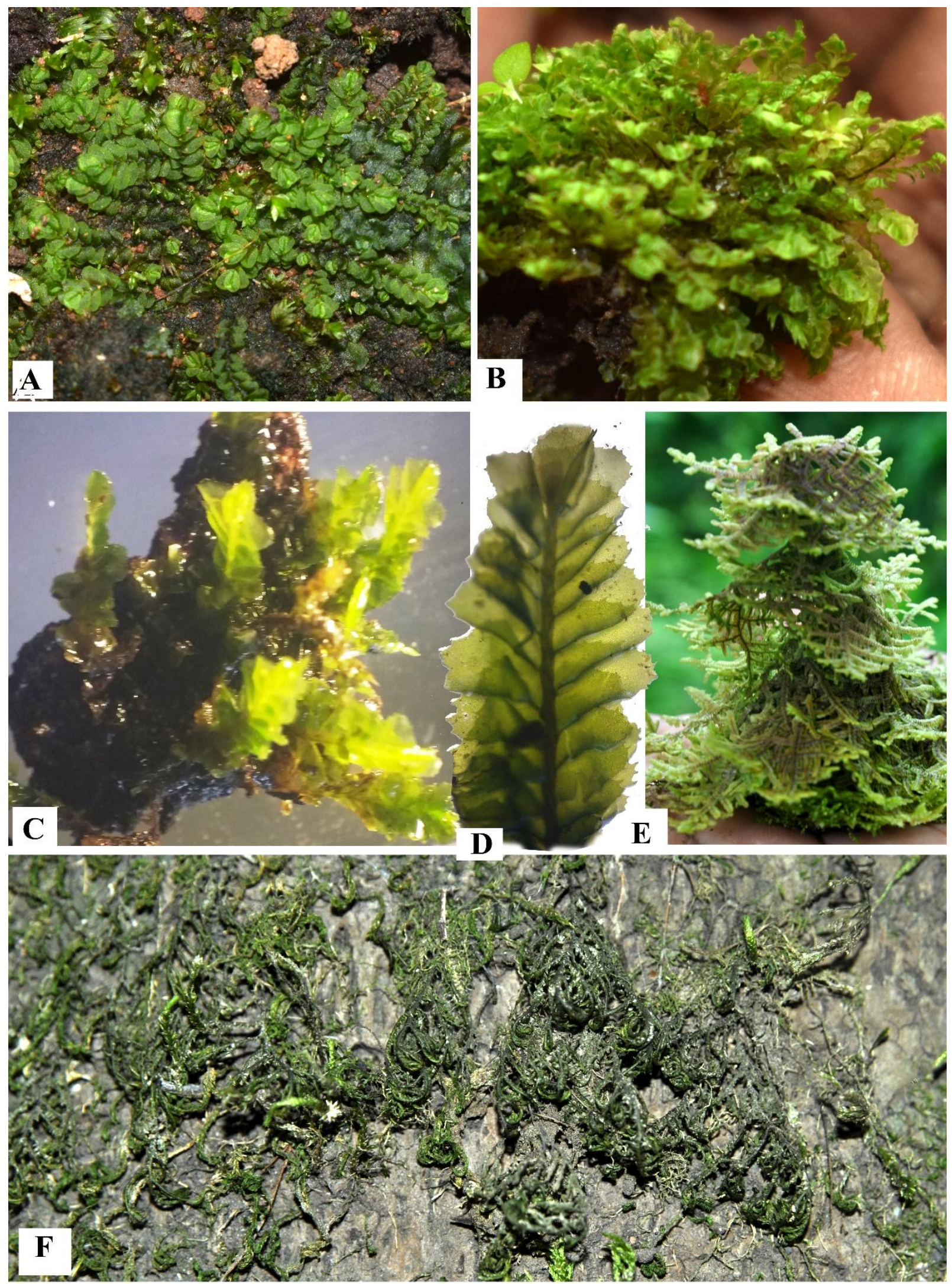

Fig. 3: A. Jungermannia macrocarpa, B. Jungermannia rubripunctata, C-D. Plagiochila beddomei, E. Plagiochila chinensis and F. Plagiochila fruticosa. 
Specimens examined: India, Andhra Pradesh, Visakhapatnam near PK Gudem, Gudem, Sambarikonda, 15 December 2017, 53943A, AS.

Cheilolejeunea krakakammae (Lindenb.) R.M. Schust., Beih. Nova Hedwigia 9: 112. 1963; Manju \& Rajesh, Bryophytes of Kerala, Liverworts. 1: 90.2017 (Fig. 2 I).

Plants leafy, yellowish-brown, up to $2 \mathrm{~cm}$ long, irregularly branched, leaves wide spreading, imbricate, lobe convex, oblong to obtuse, margin entire; marginal cells $10-17 \times 10-14 \mu \mathrm{m}$, middle cells $17-20 \times 13-17 \mu \mathrm{m}$ and basal cells $24-35 \times$ $13-20 \mu \mathrm{m}$, the first tooth an obtuse projecting cell, the second cell $1-3$ celled, free margin incurved; leaf lobule small; under leaves slightly distant to imbricate, orbicular bilobed, margin entire, base somewhat cordate, male inflorescence terminal on short lateral branches, $2-4$ pairs of bracts, inflated, half of the leaf length of the stem leaf, $1-2$ bracteoles confined to the base; female inflorescence on main branch and on lateral branches, capitate with 1 - 2 sub-floral innovations, female bracteoles large, ovate, apex bilobed, lobes obtuse.

Habitat and ecology: Corticolous, on exposed root in semi-evergreen forest, associated with Herpetineuron toccoae (Thuidiaceae).

Distribution: World: China, Japan, Nepal, Philippines and India: Kerala, Sikkim, Tamil Nadu and West Bengal.

Specimens examined: India, Andhra Pradesh, Visakhapatnam district, near PK Gudem, Gudem, Sambarikonda, 15 December 2017, 53931B, AS.

Jungermannia appressifolia Mitt., J.Proc. Linn. Soc. London, 5: 91. 1861; Amak., J. Hattori Bot. Lab. 30: 187. 1967. J. decolyana Schiffn. ex. Steph., Ibid. 6: 85. 1917; Solenostoma gollanii Steph., Sp. Hepat., 6: 81. 1917; J. monticola var. reniformis S. Hatt., J. Hattori Bot. Lab. 3: 8. 1950; J. pseudodecolyana Amak., J. Hattori Bot. Lab. 30.188.1967; J. kinabalensis Amak., J. Hattori Bot. Lab. 32: 126. 1969; Manju, Eco-sys. stud. on bryophytes of Wayanad $109-110.2005$ (Fig. 2 J).

Plants leafy, closely appressed to stem, yellowish-green when young, dark brown to blackish-green when mature, stems up to $3 \mathrm{~cm}$ long, in tufts; stem erect, branches few at the tip, innovating; rhizoids throughout the stem, numerous below, purple; leaves imbricate, rotund, sinuately inserted, decurrent on both sides, obliquely spreading, dried laterally appressed to the stem, up to $1 \times 0.7 \mathrm{~mm}$; leaf marginal cells $20-22 \times 10$ $-12 \mu \mathrm{m}$, leaf middle cells $42-48 \times 30-35 \mu \mathrm{m}$, walls thin, cuticle smooth; dioecious, perianth pyriform when young; female bracts one pair, similar to the stem leaves.

Habitat and ecology: Rupicolous on moist rocks, in moist deciduous forests, mostly monodominant or sometimes associated with other leafy liverworts.

Distribution: World: China, Japan and India: Kerala, Sikkim, Uttarakhand and Uttaranchal.

Specimens examined: India, Andhra Pradesh, Visakhapatnam District, Galikonda, near view point, 21 October 2018, 55199B, AS; Chittoor District, Near Talakona, hill top of Nelakona, Dongalabanda, 12 November 2018, 55258 \& 55259, AS.

Jungermannia macrocarpa Stephani, Sp. Hepat. 6: 87. 1917; Manju, Eco-systematic studies on bryophytes of Wayanad 110. 2005 (Fig. 3 A).

Plants leafy, light yellowish-brown, often tinged with red, stem up to $1 \mathrm{~cm}$, flaccid; stem fleshy, simple, erect, rarely with small lateral branches; rhizoids numerous, purple; leaves imbricate, widely inserted, more or less concave, $1-1.2 \times 1.2-2 \mathrm{~mm}$, leaf insertion wide and oblique; leaf marginal and middle cells $30-50 \times 20-$ $35 \mu \mathrm{m}$, leaf basal cells $45-65 \times 25-38 \mu \mathrm{m}$, walls thin, trigones distinct.

Habitat and ecology: Corticolous, on exposed roots along Gundlakamm River.

Distribution: World: China, East Nepal and India: Eastern Himalayas, Kerala and West Bengal.

Specimens examined: India, Andhra Pradesh, Prakasam District, Nallamalais, Gundlabrahmeswaram WLS., near Isukagundam, Gundlakamma river, 03 August 2017, 53532, BR \& AS.

Jungermannia rubripunctata (S. Hatt.) Amakawa, J. Hattori, Bot. Lab. 22. 38. 1960; Plectocolea rubripunctata S. Hatt. J. Hattori Bot. Lab. 3: 41. f. 36 1948; Manju \& Rajesh, Bryophytes of Kerala, 1: 76. 2017 (Fig. 3 B). 
Plants leafy, light yellow to purple green, prostrate to ascending, simple, up to $2 \mathrm{~cm}$ long, rarely branched, rhizoids purple colored, numerous on postical margin along stem, cortical and medullary cells thin walled; leaves ovate to subquadrate, distinct to contiguous, alternate apex rounded to slightly concave and reflexed marginal cells $22-25 \mu \mathrm{m}$ in diameters, slightly thick walled and minutely trigonous, median cells thin walled, trigones absent, basal cells $25-35 \times 50-70$ $\mu \mathrm{m}$, minute trigonous, thin walled. Sporophytes not seen.

Habitat and ecology: Terricolous, in earth cuttings in moist, cool, deep shady places of moist deciduous forests, associated with Notothylas levieri (Notothylidaceae).

Distribution: World: China, Japan, Nepal and India: Kerala, Meghalaya, Sikkim and West Bengal.

Specimens examined: India, Andhra Pradesh, Prakasam District, Nallamalais, Gundlabrahmeswaram WLS., near Isukagundam section, Anjaneyuni Borikalu beat, 04 August 2017, 53539, BR \& AS; Near Obulesuni penta, Bapanakonda, 05 August 2017, BR \& AS; Visakhapatnam District, Galikonda, near view point, 21 October 2018, 55199A, AS; Chittoor District, hill top of Talakona, near Sirodroni theertham, 12 November 2018, 55263C, AS.

Plagiochila beddomei Steph., Bull. Herb. Boissier, Ser. 2, 3: 876. 1903 \& Sp. Hept. 2: 361. 1903; So, Syst. Bot. Monogr. 60: 149. 2001. P. humicola Steph., Sp. Hepat. 6: 168. 1918; Manju, Eco-syst. Stud. on bryophytes of Wayanad 99. 2005 (Fig. 3 C-D).

Plants leafy, corticolous, dark brown, $4-6 \mathrm{~cm} \times 4-5$ $\mathrm{mm}$, rarely branched, rhizoids infrequent; leaves imbricate, obliquely inserted, broadly ovate, $2-2.6 \times$ $1.5-2.6 \mathrm{~mm}$, leaf base long decurrent, apex broadly truncate, irregularly $7-11$ toothed, ventral margins arched, overlap with opposite leaf base; terminal cell elongated, $50 \mu \mathrm{m}$ long, median cells $35-40 \mu \mathrm{m}$ long, basal cells $50-65 \mu \mathrm{m}$ long, trigones large, nodulose. Under leaves vestigial, paraphyllia broad; gynoecia on apical shoot, bracts similar to stem leaves, spinosedentate throughout.

Habitat and ecology: Corticolous on old tree trunks in moist shady places, associated with other pleurocarpous mosses.

Distribution: World: China, Thailand and India: Kerala, Sikkim and Tamil Nadu.

Specimens examined: India, Andhra Pradesh, Chittoor District, Horsley hills, near Krishna temple, 23 August 2016, 51612A, BR \& AS.

Plagiochila chinensis Stephani, Mem. Soc. Sci. Nat. Cherbourg 29: 223. 1894; S.C. Srivastava and Dixit, Geophytology 25: 92. 1996; So, Syst. Bot. Monogr. 60: 179: 2001; P. simplex var. pansifolia C. Massari, Mem. Accad. Agric. Verona, Ser. 3: 73(2): 12. 1897; $P$. hokinesis Steph., Bull. Herb. Biossier, ser. 2, 3: 116. 1903 \& Sp. Hepat. 2: 296. 1903; P. maireana Steph., Sp. Hepat. 6: 185. 1921; P. tongtschuana Steph., ibid. 6: 232. 1921; P. wilsoniana Steph., ibid. 6: 242. 1921; $P$. irrigata Herzog in Handel- Mauetii, Symb. Sin. 5: 18. 1930; Manju, Eco-syst. Stud. on bryophytes of Wayanad 100. 2005 (Fig. 3 E).

Plants leafy, corticolous, greenish-brown, stem up to 3 $\mathrm{cm}$ long, sparingly branched, branches, exclusively lateral intercalary, rhizoids scanty on basal part of shoot; leaves oblong-ovate, up to $1.5 \times 1 \mathrm{~mm}$, dorsal margin recurved, base long-decurrent, apex subtruncate, $15-25$ teeth per leaf, $3-7$ cells long, $1-2$ cells wide at base; terminal cells of leaves elongated, 50 $\mu \mathrm{m}$ long, basal cells $50-60 \mu \mathrm{m}$ long, trigones distinct, small. Under-leaves vestigial, paraphyllia absent; androecia terminal as well as intercalary, gynoecia terminal on main shoot, bracts broadly ovate, perianth long, irregularly toothed.

Habitat and ecology: Corticolous, on Caryota urens; associated with other pleurocarpous mosses.

Distribution: World: Bhutan, China, Nepal, Pakistan, Thailand, Vietnam and India: Kerala, Tamil Nadu, Uttar Pradesh and Western Himalaya.

Specimens examined: India, Andhra Pradesh Visakhapatnam District, hill top of P K Gudem, 15 December 2017, 53938B, AS.

Plagiochila fruticosa Mitt., J. Proc. Linn. Soc., Bot. 5: 94. 1860; So, Syst. Bot. Monogr. 60: 66. 2001; P. bipinnata Steph., Sp. Hepat. 6: 131. 1918. P. tonosa 
Steph., ibid. 6: 227. 1921; Manju, Eco-syst. stud. on bryophytes of Wayanad 103. 2005 (Fig. 3 F).

Plants leafy, greenish-brown, glossy, stem $3-10 \mathrm{~cm} \times$ $2.1-2.6 \mathrm{~mm}$, rhizome long creeping; leaves ovate to triangular, stem leaves $1.5-1.7 \times 0.5-0.8 \mathrm{~mm}$, branch leaves $1-1.2 \times 0.4-0.5 \mathrm{~mm}$, dorsal-margin slightly recurved, base long, decurrent, $6-8$ teeth per leaf, $4-6$ cells long, $4-6$ cells wide at base; terminal cells $30 \mu \mathrm{m}$ long, median cells $25-30 \mu \mathrm{m}$ long, basal cells $45 \mu \mathrm{m}$ long, trigones small. Under-leaves vestigial, paraphyllia absent; androecia intercalary, gynoecia terminal on branches, bracts irregularly toothed, perianth triangular, campanulate, highly dentate.

Habitat and ecology: Corticolous, on Caryota urens, associated with other pleurocarpous mosses.

Distribution: World: Bhutan, China, Japan, Philippines, Thailand and India: Assam, Himalaya, Kerala, Sikkim and West Bengal.

Specimens examined: India, Andhra Pradesh, Visakhapatnam District, hill top of P K Gudem, 15 Dec. 2017, 53635B, AS.

\section{Conflict of interest statement}

Authors declare that they have no conflict of interest.

\section{Acknowledgement}

We thank Andhra Pradesh Forest Department for permission to field works and colleagues of Biodiversity and Conservation Division Laboratory, Department of Botany, Sri Krishnadevaraya University.

\section{References}

Asthana, A. K., Srivastava, P., 2020. Riccia bolivinsis Jovet-Ast, New to Asia from India with distribution beyond South America, The National Academy of Science Letters.

Bapna, K. R., Kachroo, P., 2000. Hepaticology in India, Himanshu Publications, Udaipur, New Delhi. 2: pp. 413-415.

Dandotiya, D.H., Govindapyari, S. S., Uniyal, P.L., 2011. Check list of the Bryophytes of India. Arch. Bryol., 88: 1-126.

Forest Survey of India (FSI), 2019. India State of Forest Report, Ministry of environment and climate change, Dehra Dun, ed 16: 1-24.

Sandya Rani, S., Sowghandhika, M., Nagesh, K.S., Suseela, B., Pullaiah, T., 2014. Bryophytes of Andhra Pradesh. Bishen Singh Mahendra Pal Singh, Dehradun, pp. iii+279.

Sreenath, A., Ravi Prasad Rao, B., 2019. Riccia cavernosa Hoffm. (Ricciaceae, Marchantiales, Hepaticopsida) an addition to Bryoflora of South India. Plant Sci. Res., 41(1\&2): 55-57.

Sreenath, A., Ravi Prasad Rao, B., 2020. Twelve Bryophyte genera, new distributional records to Andhra Pradesh, India. J. Plant Dev. Sci., 12(3): 123-133.

Sreenath, A., Anjaneyulu, P., Nagesh, S.M., Ravi Prasad Rao, B., 2020. Addition of two species of Riccia (Mich.) L. (R. bifurca Hoffm. and $R$. subbifurca Warsnst. ex Croz) to Bryoflora of India, Plant Sci. Res., 42(1\&2): 1-3.

Sreenath, A., Anjaneyulu, P., Nagesh, S.M., Anil Kumar, M., Ravi Prasad Rao, B., 2020. Eight families of Bryophytes as new distributional records for Andhra Pradesh, India. Trop. Plant Res., 7(3): 565-572.

\section{How to cite this article:}

Sreenath, A., Ravi Prasad Rao, B., 2021. Ten species of liverworts, addition to Bryoflora of Andhra Pradesh, India. Int. J. Curr. Res. Biosci. Plant Biol., 8(7): 29-36. doi: https://doi.org/10.20546/ijcrbp.2021.807.005 